REVISTA 92.1

Revista RELACIONES INTERNACIONALES

Escuela de Relaciones Internacionales.

Universidad Nacional, Costa Rica.

N. ${ }^{\circ} 92.1$ • Enero-Junio de 2019

doi: https://doi.org/10.15359/ri.92-1.1

Pp. 13-33

\title{
LA CRISIS DE LA OMC Y EL TRILEMA DE LAS NEGOCIACIONES COMERCIALES INTERNACIONALES
}

\author{
WTO'S CRISIS AND THE TRILEMMA OF INTERNATIONAL \\ TRADE NEGOTIATIONS
}

\author{
Julieta Zelicovich ${ }^{1}$ \\ ORCID: 0000-0002-8524-9002
}

\section{RESUMEN:}

El objetivo del documento es analizar la crisis que enfrenta la Organización Mundial del Comercio (OMC) para ser un foro para las negociaciones entre sus miembros. La hipótesis es que, en el contexto de una "crisis de la globalización", se presenta un trilema en la función negociadora de la OMC. En este trilema, tres vértices, cada uno de los cuales designa una característica del proceso de negociación, no se pueden articular al mismo tiempo sino en pares, dando lugar a tres escenarios: bloqueo de las negociaciones, acuerdos light y acuerdos plurilaterales. El artículo sigue un diseño metodológico cualitativo. Se basa en una amplia revisión bibliográfica y en el análisis documental. La figura del trilema, como una forma de abordar la crisis de la $\mathrm{OMC}$, permite comprender que esta es un fenómeno intrínseco a la configuración actual del orden internacional. Además, las conclusiones apuntan al hecho de que las reformas institucionales pueden resultar un abordaje insuficiente del problema, ya que la superación de esta situación requiere de decisiones políticas.

Palabras clave: Negociaciones comerciales; Organización Mundial de Comercio; trilema; legitimidad; profundidad; efectividad.

\begin{abstract}
:
The aim of the paper is to analyse the crisis that the World Trade Organization faces for being a forum for the negotiations among its members. The hypothesis is that in the context of a "globalization backlash", a trilemma is presented in the negotiating function of the WTO. In this trilemma, the three vertices, each of which designate a feature of the negotiation process, cannot be articulated at the same time, but in pairs, in three scenarios: blockade of the negotiations, light agreements, and plurilaterals. The paper follows a qualitative methodological design. It is based on a large literature review and documental analysis. The figure of the trilemma, as a way of to tackle the WTO's crisis helps to realize that it is a phenomenon that is intrinsic to the current configuration of the international order. In addition, conclusions point to the fact that institutional reforms may result in an incomplete approach to the problem, since the overcoming of this situation needs of political decisions.
\end{abstract}

Keywords: trade negotiations; World Trade Organization; Trilemma; Legitimacy; Depth; Effectiveness.

1 Doctora en Relaciones Internacionales. Profesora de la Facultad de Ciencia Política y Relaciones Internacionales de la Universidad Nacional de Rosario, Argentina. Investigadora del Consejo Nacional de Investigaciones Científicas y Técnicas (CONICET). Correo electrónico: julieta.zelicovich@, fcpolit.unr.edu.ar 


\section{Introducción}

La parálisis de las negociaciones de Ronda Doha ha sido un elemento central de las críticas a la Organización Mundial de Comercio (OMC) durante la última década. Recurrentemente se señala que se trata de una institución que no tiene capacidad para cumplir sus metas y que se encuentra permanentemente en crisis. Este trabajo plantea como objetivo general analizar los componentes de dicha crisis, en el contexto de la llamada "crisis de globalización". El argumento central es que existe una crisis de la función negociadora de la OMC y que, en el seno de dicha función, se presenta un trilema entre los componentes del proceso de negociación, que no pueden articularse de forma simultánea, sino de a pares. El primero de los vértices es el de la profundidad de los acuerdos negociados, referidos a la capacidad de la OMC de abordar los problemas efectivamente sustantivos de las relaciones comerciales internacionales. El segundo es el de la efectividad, entendida como la habilidad de este foro multilateral de encauzar el proceso de negociaciones entre sus miembros. El tercer vértice es el de la legitimidad de carácter multilateral del proceso de negociaciones, lo que implica cumplir con los principios de la OMC y las expectativas sociales en torno a sus resultados.

El trabajo adopta una perspectiva teórica institucionalista. Su diseño metodológico se basa en la revisión bibliográfica y en el análisis de documentos. Busca contribuir en la comprensión de la problemática que atraviesa la OMC articulando la sistematización y análisis de la bibliografía existente con el estudio de los procesos negociadores que tienen lugar dentro de la "crisis". Con ello, se enmarca dentro de los estudios que desde las relaciones internacionales se enfocan en la tensión entre Estado e instituciones internacionales.

En la próxima sección se delimitan los componentes de la crisis de la OMC y las características salientes del contexto en el cual esta tiene lugar. Seguidamente se analizan los componentes del trilema, considerando sus fundamentos teóricos y metodológicos, a la vez que se presentan los escenarios que surgen de las distintas combinaciones de profundidad-legitimidad-eficacia en el marco contemporáneo. Por último, se presentan algunas reflexiones situadas en la coyuntura actual y las concusiones finales del trabajo. 


\section{La evolución de las negociaciones comerciales multilaterales entre la Conferencia Ministerial de Doha y la de Buenos Aires}

Desde que fue creada en 1995, y a lo largo de más de 20 años, la OMC ha contribuido a orientar la conducta de los Estados miembro en sus relaciones comerciales internacionales, desarrollando con éxito tres de las cuatro funciones establecidas en su tratado de creación. Ha administrado con bastante solvencia los acuerdos comerciales multilaterales, ha bregado por la transparencia en el sistema de comercio multilateral, a través del Mecanismo de examen de políticas comerciales, y ha implementado el Entendimiento de solución de diferencias. No obstante, no ha logrado resolver procesos negociadores semejantes a los del período 1947-1995, lo cual ha opacado el balance del desempeño de la institución (Vangrasstek, 2013). La Ronda Doha, lanzada en septiembre de 2001, luego de la experiencia fallida de Seattle en 1999, no ha conducido a resultados en más de 17 años de negociación, convirtiéndose en un grillete con el que carga la OMC.

La Ronda Doha supuso un verdadero test-case para la organización. Con más de 20 temas en agenda, para debatir entre -inicialmente- 142 países, y con énfasis en las cuestiones de desarrollo, significaba la negociación comercial más ambiciosa de la historia. Una serie singular de acontecimientos políticos y económicos había dado lugar al consenso necesario para lanzar el paquete de Doha (Bayne, 2005; Rolland, 2010; Steinberg, 2007), pero precisamente por su anclaje coyuntural, prontamente las negociaciones cayeron en sucesivos estancamientos.

Para el año 2002 las negociaciones se centraron en reuniones informales; seguidamente, en 2003, la Conferencia Ministerial se suspendió anticipadamente debido a las divergencias en torno a los temas de Singapur (comercio e inversiones, comercio y política de competencia, transparencia de la contratación pública y facilitación del comercio), y el enfrentamiento por el tema agrícola. En 2004 se obtienen compromisos sobre la eventual eliminación de las subvenciones a las exportaciones agrícolas, la exclusión de tres de los temas de Singapur y el uso de una formula no lineal para las negociaciones de bienes industriales, lo cual se ralentizó tras la Conferencia de Hong Kong (2005) (Zelicovich, 2017).

En 2006 y 2007 se suspendieron las negociaciones en tres ocasiones, y quedó sin realizarse la Conferencia Ministerial de 2007. Entre 2009 y 2011 la Conferencia Ministerial se reunió, aunque actuó sin avanzar sobre el Programa de Doha. Finalmente, tras varios años de parálisis, en la Conferencia de Bali como en la 
de Nairobi se lograron resultados: en 2013, mediante el Acuerdo de Facilitación de Comercio; y en 2015, con nuevos compromisos en materia de competencia a las exportaciones agrícolas. Más recientemente, en Buenos Aires (2017), no se logró alcanzar una Declaración Final, y se vuelve al estancamiento de la Ronda Doha (Zelicovich, 2017).

La bibliografía especializada en las negociaciones multilaterales de comercio ha buscado explicar las causas de esta parálisis. Un compendio de los obstáculos y dificultades que afectan en el siglo XXI el ejercicio de la función de la $\mathrm{OMC}$, de ser un foro para las negociaciones entre sus miembros, podría resumirse en al menos tres aspectos:

El primero remite a las características institucionales y de procedimiento: la (mala) adaptación del proceso de toma de decisión a los cambios en la membresía de la OMC; en particular, la incompatibilidad entre el crecimiento en la cantidad de miembros con el mantenimiento del consenso y del principio del todo único o "single undertaking" como principales mecanismos en la toma de decisiones (Low, 2011; Rolland, 2010; Steinberg, 2002). Asimismo, se han identificado como causas institucionales o procedimentales del bloqueo, el surgimiento de coaliciones negociadoras de los países en desarrollo, que han dificultado a los países centrales imponer los acuerdos como sucedía en las Rondas del Acuerdo General de Comercio y Aranceles (GATT), cambiando así la lógica de poder dentro de la OMC (Narlikar, 2005; Steinberg, 2007).

El segundo núcleo de argumentos se centra en los aspectos materiales. Se señala la creciente complejidad en la naturaleza de los temas negociados. Por un lado, debido a que la agenda involucra temas que son "problemas de cooperación de segundo orden" (Hale, Held and Young, 2013). Por el otro lado, porque los países han adoptado conductas cada vez más mercantilistas para tratar estos temas, de por sí complejos (Vangrasstek, 2013). Simultáneamente, factores externos a las negociaciones, como la variación en los precios de los commodities o el estallido de la crisis financieras internacionales, han afectado la importancia del acuerdo y la voluntad de los países para cooperar entre sí (Bellman y Hepburn, 2017; Zelicovich, 2017).

En tercer lugar, identificamos argumentos centrados en los aspectos políticos del bloqueo de las negociaciones de la Ronda de Doha. En ellos se alude a la falta de liderazgo a nivel multilateral (Rodrik, 2017), el empoderamiento político y económico de los países emergentes (Heldt, 2017), y el crecimiento de los incentivos para negociar fuera del régimen multilateral, a partir del desarrollo de las negociaciones mega-regionales (Baldwin, 2016; Bouzas y Zelicovich, 2014). 
En los últimos dos años, en el marco de lo que se ha denominado "crisis de la globalización" (Ikenberry, 2017; Primo Braga, 2017; Sanahuja, 2018) o "retraimiento de la globalización" - "globalization backlash"- (Hays, 2017; Zurn, 2018), estos factores se articularon con otras transformaciones que estaban teniendo lugar principalmente en los países desarrollados y que impactaron con fuerza en el proceso de negociaciones de la OMC. Ha habido una expansión de las críticas respecto de los efectos negativos de la globalización, que, a su vez, se ha canalizado hacia la elección de gobiernos de tinte nacionalistas, menos propensos a participar de instancias multilaterales (Baldwin, 2017; Rodrik, 2017; Sanahuja, 2016). La política comercial ha ganado centralidad en las campañas electorales y la propensión a la cooperación internacional en el área del comercio en países como Estados Unidos e Inglaterra se ha reducido.

La implementación de la política del "America First" por parte de la administración Trump ha socavado los pilares del orden liberal internacional (Ikenberry, 2017), al rechazar este las instituciones multilaterales y la reciprocidad difusa como principio de acción, para adoptar en su lugar lógicas mercantilistas, una reciprocidad de tipo específica, y la priorización de los vínculos bilaterales (Primo Braga, 2017). La "guerra comercial" de esta administración con China ha planteado, asimismo, un serio desafío a la preservación de un sistema de comercio basado en reglas.

Entendemos que el bloqueo de las negociaciones no se explica por uno solo de estos factores, sino por su combinación. En la próxima sección se propone poner en relación estos distintos argumentos (institucionales, procedimentales, materiales, políticos y contextuales) con la figura del trilema.

\section{EI trilema en la función negociadora de la OMC}

Conforme con el enfoque institucionalista de las relaciones internacionales, las organizaciones internacionales, como la $\mathrm{OMC}$, tienen que tener capacidad de regir la conducta de los Estados, facilitando la concreción de acuerdos cooperativos entre los gobiernos. Esa capacidad de facilitar los acuerdos reside en su actuación como modificadora de las expectativas de conductas de los Estados y como orientadora de su interacción, a partir de su capacidad de alterar los costos relativos de las transacciones, proveer información y generar expectativas de relacionamiento a mayor plazo (Keohane, 1984; Martin, 1992).

La función de la OMC de ser foro para las negociaciones entre sus miembros (y su crisis) puede interpretarse a partir de este marco teórico. ¿De qué manera las negociaciones en el marco de esta organización pueden facilitar conductas 
cooperativas entre las partes? ¿Qué requisitos tienen que reunir los acuerdos para poder concretarse? Y, conforme lo expuesto en el apartado anterior: ¿Por qué ello no acontece en el contexto actual? Se trata de indagar en la tensión que existe en el vínculo entre el Estado y las instituciones internacionales (Stone, 2009).

El argumento central de este trabajo es que la tensión que se presenta en las negociaciones comerciales internacionales en el siglo XXI puede ilustrarse con un trilema ${ }^{2}$, en el que, como se señaló supra, interactúan: la profundidad de los temas negociados; la legitimidad con la que cuenta el proceso negociador, y la efectividad con la que se lleva a cabo.

El trilema es contingente, coyuntural, por oposición a estructural, en las negociaciones multilaterales; y se correlaciona con la crisis del orden liberal internacional. Durante el período del GATT, los tres componentes mencionados anteriormente pudieron combinarse adecuadamente, lo que llevó al logro de ocho rondas de negociación exitosas. Sin embargo, en el contexto actual, estos tres componentes funcionan de forma armoniosa solo en pares (Figura 1).

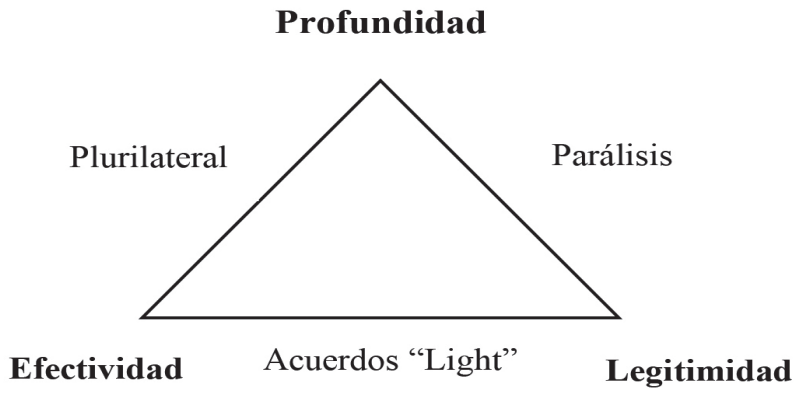

Figura 1. Trilema de las negociaciones comerciales internacionales en el siglo XXI. Elaboración propia.

\section{Los vértices}

Dentro del trilema, el primero de los vértices es la profundidad de la materia negociada, en términos de alcance o sustancia. Las negociaciones internacionales como mecanismos de cooperación versan sobre aquello que los Estados identi-

2 Esta lógica ha sido utilizada en más de una oportunidad para ilustrar las tensiones de la globalización, destacándose el trilema de Obstfeld y Taylor (1998) respecto de la articulación de apertura de la cuenta de capital, independencia de la política monetaria y mantenimiento de tipo de cambio fijo, y el trilema de Rodrik (2000) entre integración económica mundial, Estado-Nación y democracia. 
fican como área de problema: la existencia de una potencial discordia a la vez que intereses comunes (Keohane, 1988). La profundidad alude a la proximidad que lo negociado tiene con dicha área de problema: dentro de la $\mathrm{OMC}$, la capacidad de lo negociado de orientar las conductas del comercio; de que los temas y compromisos que se acuerdan sirvan para ordenar los intercambios de bienes y servicios realmente existentes. En este sentido, los acuerdos negociados deberían servir para brindar previsibilidad y transparencia a aquellas cuestiones que se presentan como áreas de conflicto en el comercio internacional y en las prácticas de los Estados en sus relaciones comerciales internacionales.

Existen múltiples maneras de entender la profundidad: en razón de su cobertura sectorial -tanto en términos de posiciones arancelarias consideradas como en términos de sectores económicos-; en razón del nivel de liberalización -en cuanto a la ambición de los compromisos involucrados-; y en términos de cobertura de provisional, que considera si se mencionan ciertos aspectos de la integración (Damuri, 2012; Hofmann, Osnago y Ruta, 2017). Gilpin (2001) estipula que el contenido de un régimen internacional se determina en función de los factores tecnológicos, económicos y políticos imperantes. Y conforme con estudios como el de Mattoo y Subramanian (2008), existe una relación entre la profundidad de la agenda, la relevancia que adquiere la negociación y el involucramiento de actores en esta. Para estos autores, el problema de la Ronda Doha es que el paquete de temas no es lo suficientemente profundo para el interés negociador (de grupos claves).

En consecuencia, lo que debe incluir un acuerdo para ser considerado profundo cambia con el tiempo a medida que se modifican las prácticas comerciales, tal como lo muestra la evolución de la agenda del GATT y la de los acuerdos comerciales preferenciales. Si bien tradicionalmente se refiere a la profundidad a partir de la inclusión de temas OMCx y OMC+ (OMC, 2011), entendemos que el grado de profundidad de un acuerdo debe entenderse de una manera más amplia -en razón de la bibliografía referida- y aproximarse a partir de considerar su impacto en las políticas que originan las tensiones en las relaciones comerciales internacionales y en los flujos de intercambio resultantes.

En el siglo XXI, tanto la naturaleza del comercio, ahora cada vez más involucrada en las cadenas globales de valor, como las características de la política comercial, que se han adaptado al espacio de maniobra que han dejado las negociaciones internacionales anteriores, resultan mucho más complejas que antes (Damuri, 2012; Hale, et. al., 2013). Las negociaciones de la OMC incluyen actualmente dos tipos de agendas temáticas que pueden ser consideradas profundas, por sus impactos potenciales sobre el comercio y la política comercial. 
Por un lado, se contempla la agenda de los temas pendientes de la Ronda Doha, en la que se incluyen temas tradicionales como los aranceles de bienes industriales y agrícolas, las normas de antidumping, los subsidios agrícolas, las reglamentaciones relacionadas a pesca, etc. Por otro, se hace referencia a la agenda de "nuevos temas", los que responden a las necesidades del "regionalismo del siglo XXI" (Baldwin, 2011) donde se engloban cuatro cuestiones prioritarias: política de competencia, movimientos de capital, propiedad intelectual e inversiones. Se incluye también en esta agenda la facilitación del comercio, el comercio electrónico, y las cuestiones de medio ambiente y de políticas laborales (Damuri, 2012; Hofmann, Osnago y Ruta, 2017; OMC, 2011).

Ninguna de estas dos agendas tiene un "camino fácil" en el proceso de negociación. La agenda de los temas pendientes de la Ronda Doha, por estar centrada en sectores internos sensibles es el remanente de los acuerdos y procesos de liberalización de las rondas anteriores. Se trata de aspectos en los que la propensión a la cooperación internacional es menor y los acuerdos son más difíciles de alcanzar. La agenda de los "nuevos temas", por su parte, resulta difícil de abordar, debido a sus especificidades técnicas y políticas: son temas en los que hay múltiples formas posibles de llevar a cabo la cooperación internacional, resulta menos claro que en materia de aranceles cuáles son los espacios de acuerdos deseables y posibles.

Esto son temas que, además, tienen una mayor intrusión en las practicas nacionales, tradicionalmente no sujetas a supervisión nacional (Bouzas y Zelicovich, 2014) y que -como se documenta en Barton, Goldstein, Josling y Steinberg (2008)- generan diferentes tipos de costos de ajuste, como la creación de nuevas agencias gubernamentales o involucran procesos jurídico-administrativos complejos tanto para su sanción como para su implementación. El proceso de implementación de Acuerdo sobre Facilitación de Comercio permite ilustrar esta referencia (OECD y OMC, 2015).

La efectividad, el segundo vértice, remite a la capacidad de la OMC para producir resultados. Esto es, acuerdos entre los países miembros, a partir del cumplimiento de su función de ser foro para las negociaciones entre los Estados que la conforman. Una negociación efectiva es aquella que se concluye en un período razonable de tiempo, alcanzando un acuerdo mutuamente aceptable entre las partes; y un acuerdo de tales características es el que las partes perciben como superador del status quo previo a las negociaciones, o bien del status quo sin negociaciones. Cecilia Albin define la efectividad de un proceso de negociación como "la capacidad para llegar a un acuerdo oportuno, que resuelva o establezca los aspectos importantes [del vínculo] y que sea implementada por la cantidad suficiente de partes para cumplir con sus objetivos" (Albin, 2012, p. 2). 
Dentro de las negociaciones OMC, la efectividad depende de tres aspectos complementarios. Por un lado, de la posibilidad de establecer vinculaciones entre cuestiones; algo que conforme a los estudios es uno de los mecanismos centrales para alcanzar una zona de acuerdo posible en un proceso de negociaciones (Hoekman and Kostecki, 2001). Por otro lado, es reconocido que los aspectos institucionales formales y procedimentales pueden incrementar la efectividad en un proceso de negociaciones. Se plantea que la OMC debería tener los recursos institucionales suficientes para guiar las negociaciones entre miembros, ayudándolos a identificar y establecer esas posibles áreas de acuerdo (Steger, 2009). En tercer lugar, la existencia de liderazgos fuertes - que puedan proveer incentivos, pagos laterales, sanciones y amenazas en el proceso de negociación- es clave para la efectividad ${ }^{3}$. En otros términos: "el proceso de negociación depende, en gran medida, de si un acuerdo sobre un asunto es realmente importante para los jugadores principales" (Hoekman and Kostecki, 2001, p. 80).

Las negociaciones en la OMC (que van desde el establecimiento de la agenda, la aprobación de los mandatos, la definición de los intercambios y adecuaciones de normativas, su aprobación e implementación) se rigen por una serie de principios que regulan la toma de decisiones desde la época del GATT: el consenso y la reciprocidad. Las negociaciones suelen llevarse a cabo en el formato de "rondas" cuyo mandato queda establecido por la Conferencia Ministerial. Asimismo, se incluye la práctica de negociaciones informales, como las instancias de negociaciones reducidas (Wolfe, 2004).

Este esquema decisorio no ha logrado, sin embargo, ser eficaz en la Ronda Doha. Tanto los cambios en el balance de poder global como el uso incremental del veto entre participantes (como correlato de la regla del consenso), y la vigencia del principio de negociación como un paquete único han obstaculizado el acuerdo de Doha. El recurso a las coaliciones negociadoras - tanto "tradicionales" como "de nuevo tipo" (Narlikar, 2005)- ha empoderado a los países en desarrollo, a la vez que ha provocado profundos cambios en las dinámicas de negociación de la $\mathrm{OMC}$, en especial en lo que hace a los espacios informales de negociación, que desde la etapa del GATT marcaban el tiempo del proceso de acuerdos.

Por su parte, la "crisis de la globalización” ha incrementado los desafíos del vértice de la efectividad, en tanto que la administración Trump ha puesto en

3 Acorde con Kindleberger (1981), cuando los Estados cuentan con capacidad de influir en el sistema internacional tienen mayor posibilidad de afectar la generación de los bienes públicos y, por lo tanto, tienen un incentivo para emprender esta tarea. A lo largo de la segunda mitad del siglo XX, EEUU fue el actor que proveyó los incentivos para que el comercio mundial se ordenara en torno a una serie de pautas de conducta que permitiera que su crecimiento y expansión. 
cuestión la forma en la que las negociaciones se han llevado a cabo hasta ahora (con especial foco en la manera de abordar la reciprocidad a partir del trato especial y diferenciado), e incluso ha planteado reservas respecto de la pertinencia de la OMC para dirigir el proceso de negociaciones en curso, y ha bloqueado de manera sistemática el nombramiento de miembros para el órgano de apelaciones del mecanismo de solución de diferencias.

El tercer vértice es el de la legitimidad. Los acuerdos requieren no solo ser profundos y efectivos, sino que además necesitan ser considerados legítimos. Al respecto, Keohane sostiene que hay dos formas de considerar la legitimidad: por un lado, la legitimidad legal, cuando el proceso cumple con los estándares establecidos; por otro lado, la legitimidad sociológica, cuando el proceso es aceptado como apropiado y meritorio de ser acatado por su audiencia (Keohane, 2006). La legitimidad permite a los actores coordinar su apoyo a instituciones particulares al apelar a una voluntad común de movilizarse por razones morales, a diferencia de las razones puramente estratégicas o exclusivamente egoístas (Buchanan y Keohane, 2010).

Dentro de las discusiones de la OMC, y con especial énfasis tras la Conferencia Ministerial de Seattle, se ha hecho foco en los aspectos sociales de la legitimidad de la política comercial externa y las negociaciones multilaterales. Collier (2008), por ejemplo, se ubica en la discusión de lo que se conoce como "inputlegitimacy" e identifica una serie de aspectos en los que la OMC presenta un déficit de legitimidad: en la relación entre acuerdos internacionales y regulaciones nacionales -donde los primeros se "imponen" a los segundos a expensas de la voluntad de la ciudadanía-; también puntualiza el mantra técnico con el que se consideran estas cuestiones dentro de los procesos decisorios a nivel doméstico, lo cual los aleja de la discusión ciudadana. En cuanto a la llamada "output-legtimacy", existe un amplio consenso respecto de que los efectos de las disposiciones acordadas sobre los flujos del comercio y sobre el bienestar social contribuyen a incrementar o disminuir la legitimidad social de la propia OMC. En dicho sentido, la falta de resultados de la Ronda ha retroalimentado la crisis de legitimidad del organismo (Coldicott, 2008).

Respecto de la "calidad democrática" del proceso de negociación, la historia del GATT había estado plagada de situaciones de "minilateralismo" (Kahler, 1992) que se extendieron en la etapa de la OMC. Daemmrich (2011) plantea que fue en torno a las negociaciones para el cierre de la Ronda Uruguay que los países en desarrollo se encontraron con las reglas tácitas con las que se regían las negociaciones y que erosionaban su legitimidad: las negociaciones involucraban largas sesiones, pero las decisiones claves se tomaban en pequeños grupos de trabajo dominados por las economías desarrolladas (Daemmrich, 2011). A con- 
clusiones similares llegan Jawara and Kwa (2003) a partir de un extenso análisis documental de las negociaciones en torno al lanzamiento de la Ronda Doha. Se plantea así una vinculación estrecha entre el multilateralismo, "prácticas democráticas" y legitimidad.

El multilateralismo, en efecto, es considerado en la teoría un componente central de la legitimidad. La diversidad de intereses que presenta es una de las claves de su contribución en este aspecto (Keohane, 2006). Otras ventajas del multilateralismo en las negociaciones comerciales internacionales remiten a que, al ser comercio internacional crecientemente interdependiente y global, la armonización de la regulación del comercio necesaria para su funcionamiento solo puede alcanzarse de manera multilateral (Baldwin, 2016).

\section{Tres escenarios}

La Ronda Doha, como fue concebida en 2001, debía combinar profundidad, efectividad y legitimidad. Sin embargo, estos aspectos no pudieron ser alcanzados de forma simultánea. Como resultado, emergen tres escenarios alternativos.

El primer escenario es el que resulta de la combinación de los vértices de legitimidad y profundidad, sin alcanzar el de efectividad. Su correlato empírico es el del bloqueo de las negociaciones de Ronda Doha.

Como fue mencionado, el progreso en las negociaciones de la Agenda de Doha para el Desarrollo ha sido realmente acotado. Desde el inicio del proceso de negociaciones, los espacios de acuerdo fueron reducidos y tendieron a prevalecer estrategias de negociación confrontativas, con la regla del single undertaking. Esto condujo a la suspensión de las negociaciones en múltiples ocasiones desde 2006 en adelante.

Dentro de los temas de la agenda, la cuestión agrícola inicialmente era percibida como la que mayor potencialidad tenía de impactar en el comercio internacional y sobre el desarrollo ${ }^{4}$. El tema, no obstante, estaba condicionado por las sensibilidades que generaba en el doble enfrentamiento entre países desarrollados y países en desarrollo, y países exportadores y países importadores de

4 Los primeros estudios estimaban "en 254000 millones de dólares anuales de 1995 los beneficios de una completa liberalización del comercio y de los subsidios agrícolas, de los cuales 108000 millones recaerían en los países en desarrollo" (Steinberg, 2007, p. 90). En algunas cuestiones la relevancia de la agenda fue diluyéndose sea por cambios unilaterales en las prácticas de las políticas comerciales como por la variación en los precios de los commodities que modificó los incentivos detrás de las políticas distorsivas. 
alimentos, donde el caso más saliente fue el de EEUU con India en materia de salvaguardias o seguridad alimentaria.

En los nuevos temas, cuya relevancia para el comercio en cadenas globales de valor fue acentuándose cada vez más, las restricciones sobre el proceso negociador eran similares. Las diferencias entre los países se manifestaron en los temas Singapur, mientras que otros temas como acceso a mercados no agrícolas (AMNA) quedaron virtualmente congelados -en parte bloqueados- por la paralización de las negociaciones agrícola y las implicancias de los mecanismos de toma de decisión por consenso con "single undertaking".

De esta forma, con una agenda de temas "profundos" y conservando prácticas de negociación que, aún con un cúmulo de déficits, pretendían cumplir ciertos estándares de legitimidad multilateral, no resultaba viable identificar zonas posibles de acuerdo ni alcanzar un consenso entre las partes en un tiempo adecuado. En este escenario, el mantenimiento de una ambiciosa agenda de temas, con un proceso negociador "legítimo", en razón de la preservación del carácter multilateral y democrático de las negociaciones, conduce a espacios de acuerdo reducidos, en un contexto en el que las divergencias entre los países son elevadas y no hay un líder claro con interés de "pagar los costos" de la cooperación multilateral. El resultado es el bloqueo de las negociaciones, o bien su avance a cuentagotas.

No obstante, se argumenta que si la OMC no produce resultados, corre el riesgo de que la crisis que enfrenta en su función de ser foro para las negociaciones entre sus miembros se extienda hacia las demás, lo que la volvería una organización irrelevante para el comercio internacional. Así, una opción frente a la falta de eficacia del proceso negociador de Doha ha sido aludir a la "cosecha temprana": la posibilidad de concretar acuerdos parciales por fuera del single undertaking, contemplada en el artículo 47 de la Declaración Ministerial de Doha. Este escenario combina legitimidad democrática multilateral y eficacia del proceso negociador, pero sacrifica la profundidad, dando lugar a "acuerdos light".

Siguiendo esta alternativa, tanto en Bali (2013) como en Nairobi (2015) se presentaron resultados en la esfera multilateral. Si bien estos mismos resultan importantes para la OMC, en el contraste con la agenda pendiente de la Ronda Doha su aporte es marginal. Se trata de temas donde los costos de ajustes para las partes son menores, o bien, en los que las sensibilidades fueron reduciéndose con el tiempo a partir de otros factores externos a la negociación -por ejemplo, las subvenciones a las exportaciones agrícolas habían reducido su peso como instrumento de política comercial en Europa previamente a que se aprobara el paquete de Nairobi-. 
El escenario de legitimidad y eficacia sin profundidad produce dos tipos de reacciones. Los optimistas señalan que se trata de una buena manera de avanzar en la regulación del comercio internacional. Por ejemplo, Rodrik indica que tal es una vía apropiada para resolver las tensiones entre hiperglobalización y soberanía nacional: "una globalización inteligente, no una globalización máxima" (Rodrik, 2012, p. 21). Por su parte, Hoekman y Kostecki reconocen que acuerdos simbólicos en ocasiones pueden ser relevantes para el desarrollo político del sistema multilateral y, por ende, también tienen un valor intrínseco que va más allá de la letra escrita (2001).

Desde la perspectiva pesimista, se alerta, en cambio, sobre los riesgos de que la $\mathrm{OMC}$ solo pueda ser foro para negociaciones poco relevantes en materia de comercio mundial. Se sostiene que si la OMC no logra avanzar en el diseño de normas del "regionalismo del siglo XXI" tendría cada vez menor legitimidad, su capacidad para impulsar la cooperación internacional sería menor, a la vez que se perjudicaría la relevancia del órgano de solución de diferencias de la OMC (Baldwin, 2011; Primo Braga y Hoekman, 2017).

En esta línea pueden identificarse algunos efectos negativos recientes sobre la dinámica de negociaciones comerciales tras el arreglo de subvenciones a las exportaciones en la Conferencia Ministerial de Nairobi. Por un lado, al romper el single undertaking este resultado contribuye a consolidar un núcleo de temas en el que no se pautan vínculos de cooperación -como acceso a mercados agrícolas- (Hoekman 2014). Por otro lado, porque al haberse demorado el acuerdo sobre subvenciones a las exportaciones la forma en la que este se concretó tiene más proximidad con las políticas unilaterales de los actores poderosos que con la agenda de reclamos de muchos de los países en desarrollo (Zelicovich, 2016). Por último, porque este acuerdo debilita la cohesión entre los países en desarrollo al perderse el tema que mantenía vinculadas a las coaliciones del G20, G-33 y G-45, restándole importancia política a la agenda de estos actores (Martin y Mercurio, 2017).

El escenario de los acuerdos light se basa en el acuerdo mínimo común que preserva la legitimidad multilateral conforme los lineamientos existentes. Reconoce las restricciones en el escenario internacional para avanzar en temas significativos, y propone una agenda centrada en lo posible que, no obstante, deja a muchos Estados insatisfechos.

5 Mientras que el G33 es una coalición de países en desarrollo que exigen flexibilidad en las negociaciones agrícolas para que la apertura de mercado sea acotada. El G4, también conocido como Cotton-4, es una coalición de países de África Occidental que buscan recortes en los subsidios y aranceles del algodón. 
La tercera opción dentro del trilema es la de combinar eficacia y profundidad, sacrificando el vértice de legitimidad. Este escenario, denominado como "acuerdos plurilaterales" parte de la existencia de un trade off entre el crecimiento de la cantidad de miembros y la mayor heterogeneidad de la membresía, frente a la posibilidad de arribar a acuerdos profundos. Como resultado han emergido, de manera creciente, instancias plurilaterales y megarregionales para abordar las negociaciones sobre los temas complejos del comercio internacional con mayor eficacia, pero solo entre aquellos Estados interesados -y con capacidad para hacerlo.

Los acuerdos plurilaterales no son una novedad en las negociaciones de comercio. Fue esta la modalidad de los acuerdos de la Ronda Tokio, y también la de los de Aviación Civil, Compras Públicas, Productos Lácteos y Carnes Bovinas. Sin embargo, mientras que estos acuerdos estaban anclados en el régimen multilateral -y no lesionaban de manera sustantiva la legitimidad-, los plurilaterales que se han negociado en la segunda década del siglo XXI no guardan relación con la OMC, o lo hacen a medias, a partir de la excepción del artículo XXIV del GATT-94. El Acuerdo Transpacífico (TPP) fue hasta el 2015 el ejemplo "exitoso" de esta tendencia -luego matizado tras la retirada de EE.UU. y la firma del Acuerdo Integral y Progresivo para el TPP (CPTPP)-, que se superponía con experiencias próximas como el Acuerdo de Tecnología de la Información (ITA), o el Acuerdo sobre Comercio de Servicios (TISA), entre otros. La iniciativa más reciente -aún en etapa preliminar- es la de un acuerdo plurilateral sobre comercio electrónico.

En todos los casos se trata de acuerdos con gran volumen del comercio específico involucrado y con el desarrollo de normas que van más allá de lo pautado en OMC, tanto por los temas involucrados como por la ambición de sus compromisos.

El avance de esta opción dentro del trilema provoca una serie de efectos negativos para el sistema, dado que carece de legitimidad multilateral. Por un lado, se argumenta que los nuevos acuerdos "no multilaterales" generan una regulación asimétrica del comercio global, al crear núcleos o nodos de regulaciones normativas, donde los países en desarrollo quedan en la periferia. También se señala un incremento en la incertidumbre en el comercio global, a la vez que mayores costos para la operatoria comercial (Pauwelyn y Alschner, 2014; Rosales et al., 2013). Por otro lado, estos acuerdos plurilaterales erosionan la relevancia del mecanismo de solución de diferencias de la OMC (Baldwin, 2016). En tercer lugar, como señala Hoekman (2014), la consolidación de un conjunto de temas de baja cooperación: normas sobre las que no hay consenso ni en la esfera mul- 
tilateral ni en la plurilateral, y que quedarían virtualmente excluidas de cualquier esfuerzo de regulación.

\section{¿Puede convertirse el trilema en un "círculo virtuoso"?}

En gran parte, el (buen) desempeño de la función negociadora del GATT se debió a que existían mayores convergencias políticas en torno a la identificación de qué aspectos del comercio internacional requerían de la cooperación entre los Estados y en qué sentido debía orientarse dicha cooperación -lo que hemos llamado "profundidad"-; asimismo, existía un fuerte liderazgo de los EE.UU., que dotaba al sistema de efectividad. Por su parte, había un entendimiento más laxo de la legitimidad, y con ello de multilateralismo y de prácticas democráticas. Esta convergencia contribuía a la generación de un círculo virtuoso entre los tres pilares que analizamos en este trabajo en el marco de la función negociadora en el plano multilateral.

La heterogeneidad es, por contraste, la norma en las relaciones comerciales internacionales en el siglo XXI. Países con muy diferentes modelos y estadios de desarrollo disputan en torno a la forma de regular el comercio internacional, el cual a su vez se volvió más complejo. La falta de un liderazgo claro, así como el aumento de los gobiernos adversos a la globalización en países que tienen mayor poder de mercado e influencia en las negociaciones, hacen casi imposible esperar que el trilema pueda volver a transformarse en un circulo virtuoso. En este contexto, y asumiendo que esta crisis del orden liberal internacional presenta características que hacen plausible su extensión en el tiempo (MéndezCoto, 2016; Sanahuja, 2018), la pregunta es si es posible ajustar el trilema para que la $\mathrm{OMC}$ pueda recuperar su función como foro para las negociaciones entre sus miembros.

Si bien, dado el contexto de crisis de globalización, el trilema no puede resolverse, identificamos algunas cuestiones que pueden suavizarlo. Una cuestión con amplio tratamiento en la bibliografía especializada es la reforma de aspectos institucionales como la remoción del principio del single undertaking o la adopción de enfoques como el de los plurilaterales o el de la masa crítica, para los nuevos acuerdos (Wolfe, 2009; Vickers, 2013). Estas propuestas, sin embargo, si bien ganan en "efectividad", suponen sacrificios en "legitimidad" multilateral, y no logran contribuir de manera necesaria en la "profundidad".

Otro camino, menos frecuentemente considerado, es el de los procedimientos y prácticas, dentro del marco actual, que fue lo que hizo posible los acuer- 
dos de Bali y Nairobi. La manera en la que se organizan las negociaciones en los grupos de negociación reducidos, y cómo estos cuerpos se articulan en el foro multilateral -considerando los principios de transparencia, legitimidad e inclusión- puede contribuir a alcanzar los acuerdos. De esta forma fue que se concretaron los resultados de 2013 y 2015, con una metodología bottomup, una participación activa de los chairs de estas áreas (Neufeld, 2014), y la generación de propuestas conjuntas entre países desarrollados y en desarrollo (Zelicovich, 2017). No obstante, la reforma en los procedimientos es solo una ayuda parcial, puesto que -tal como sucedió en Bali y Nairobi, donde el apoyo de EE.UU. y Europa resultaron clave- el factor decisivo es el político y este es el que se encuentra ausente en las negociaciones comerciales, dada la crisis de globalización.

\section{Reflexiones finales}

El punto de partida de este trabajo ha sido reconocer que la crisis de la OMC está centrada en su incapacidad de llevar adelante la función de ser foro para las negociaciones entre sus miembros. Hemos sostenido que ello encuentra raíces de tipo institucionales y procedimentales; y también causas que son materiales y políticas, y que pueden ser identificadas a lo largo de los últimos 15 años. La llamada "crisis de la globalización" ha acentuado los condicionantes negativos del entorno para el avance de mecanismos de cooperación para las negociaciones comerciales internacionales, y ha conducido a la situación que plantea el trilema. En esta misma, tres componentes del proceso negociador, que en la bibliografía institucionalista se reconocen como centrales para la creación y sostenimiento de acuerdos cooperativos en el marco de regímenes internacionales, no pueden articularse de manera simultánea.

El abordaje planteado con el trilema permite apreciar que para el régimen multilateral de comercio legitimidad, profundidad y efectividad -usualmente abordados de manera individual- no son aditivos de un proceso negociador sino que conforman un todo. Si una nueva negociación en el marco de la OMC debe servir para orientar las conductas de los países miembros, desde la literatura se desprende que los acuerdos resultantes deberían poder lidiar con aquellas temáticas que se presentan como áreas de tensión en las relaciones comerciales internacionales -ser profundos- ; deberían hacerlo en un tiempo y modo que guarde relación con el problema planteado -ser efectivos- ; y tendrían que ser considerados legítimos por las partes involucradas, de manera de mediar en la tensión entre soberanía estatal e internacionalismo. Las tres condiciones, a la vez. 
Otra contribución que plantea el trilema alude a la temporalidad de la crisis. El trilema ayuda a comprender que se trata de un fenómeno inherente a la configuración actual del orden internacional. No tiene carácter estructural puesto que, en otros tramos históricos, los tres componentes estudiados funcionaron de manera armoniosa, dando lugar a diversos avances en la regulación cooperativa de las relaciones comerciales internacionales.

Por su parte, el trilema contribuye también a un análisis crítico de las propuestas de reforma circulantes entre la academia y policy makers al permitir identificar los costos que cada opción supone respecto de la totalidad del proceso negociador. La conclusión es que la mayoría de estas no logra presentar una solución de fondo a la crisis. Coincidimos con Vickers cuanto este afirma:

No existe una 'panacea procedimental' que mágicamente resuelva el espectro de diferencias sustanciales de los temas fundamentales de la agenda de Doha. ... En otras palabras, los refinamientos constitucionales y procedimentales, en el mejor de los casos, podrían hacer más fácil el camino para tomar decisiones y eventualmente concluir las negociaciones. (Vickers, 2013, p. 1)

La variable central para que legitimidad, eficacia y profundidad puedan articularse nuevamente es política: se precisan liderazgos y percepciones compartidas. Sin convergencia política no puede esperarse a corto plazo que la $\mathrm{OMC}$ se vuelva más eficiente en su proceso de negociación, mantenga profundidad en los temas negociados y gane legitimidad con carácter multilateral.

En un mundo que atraviesa una crisis de globalización, con componentes políticos, ideológicos y económicos, tal convergencia no parece factible de lograrse. El trilema manifiesta así una situación trágica para la $\mathrm{OMC}$, que debe repensar sus principios y prácticas para mantenerse como un foro capaz de orientar las negociaciones entre sus miembros, a costa de sacrificios históricos, o correr el riesgo de volverse cada vez menos significativa para las relaciones comerciales internacionales y la cooperación internacional.

\section{Referencias}

Albin, C. (2012). Improving the Effectiveness of Multilateral Trade Negotiations: A Synopsis, International Negotiation, vol. 17 No. 1, pp. 1-8.

Baldwin, R. (2011). 21st century regionalism: Filling the gap between 21st century trade and 20th century trade rules. WTO Staff Working Papers ERSD-2011- 
08, World Trade Organization (WTO), Economic Research and Statistics Division.

Baldwin, R. (2016). The World trade organization and the future of multilateralism. Journal of Economic Perspectives, 30(1), 95-116.

Baldwin, R. (2017). The great convergence. Information technology and the new globalization. Cambridge: The Belknap Press of Harvard University Press.

Barton, J. H., and Goldstein, J. L., Josling, T. E. y Steinberg, R. H. (2008). The Evolution of the Trade Regime: Politics, Law, and Economics of the GATT and the WTO, Princeton: Princeton University Press.

Bayne, N. (2005). Staying together: The G8 summit confronts the 21st Century, Burlington: Cornwall Ashgate Publishing.

Bellmann, C., and Hepburn, J. (2017). The Decline of Commodity Prices and Global Agricultural Trade Negotiations: A Game Changer? International Development Policy, Revue internationale de politique de développement [Online], artículo 8.1. doi: 10.4000/poldev.2384

Bouzas, R., and Zelicovich, J. (2014). La Organización Mundial de Comercio, los acuerdos mega-regionales y los usos estratégicos del regionalismo. Estudios de Economía Aplicada, 32(3), 963-994.

Buchanan, A., and Keohane, R. (2010). The legitimacy of Global Governance Institutions. In J. Babic, P. Bojanic (Eds.), World Governance: Do we need it, Is it possible, What could it (all) mean? Newcastle: Cambridge Scholars Publishing.

Coldicott, D. (2008). The World Trade Organization, Legitimacy and the Development Problematic. Paper presented to the PhD Workshop of the Annual Bank Conference on Development Economics (ABCDE), Cape Town, South Africa, 7-8 June 2008. Available at http://siteresources.worldbank.org/INTRAD/ Resources/DColdicott.pdf

Cottier, T. (2008). The Legitimacy of WTO Law. NCCR Trade Regulation, Working Paper 19, Swiss National Centre of Competence in Research, available at http://phase1.nccr-trade.org/images/stories/publications/IP2/The_Legitimacy_of_WTO_Law_cottier_final\%200808.pdf (Accessed 1 December 2017)

Daemmrich, A. (2011). The Evolving Basis for Legitimacy of the World Trade Organization: Dispute Settlement and the Rebalancing of Global Interests. Harvard Business School, Working Paper 12-041, available at http://www20. iadb.org/intal/catalogo/PE/2012/09920.pdf

Damuri, Y. (2012). 21 ${ }^{\text {st }}$ Century Regionalism and Production Sharing Practice. CTEI Working Papers 4, Centre for Trade and Economic Integration, The Graduate Institute, Geneva. 
Gilpin, R. (2001). Global Political Economy. Understanding the international economic order. Princeton University Press.

Hays, J. (2017). Embedded Liberalism and the Populist Backlash. University of Pittsburgh. Mimeo.

Hale, T., Held, D., and Young, K. (2013). Gridlock. Why global cooperation is falling when we need it most? Cambridge: Polity Press.

Heldt, Eugenia. (2017). Shaping Global Trade Governance Rules: New Power's Hard and Soft Strategies of influence at the WTO. European Foreign Affairs Review, 22(Special Issue), 19-36.

Hoekman, B. (2014). Supply Chains, Mega-Regionals and Multilateralism: A Road Map for the WTO. London: CERP Press.

Hoekman, B., and Kostecki, M. (2001). The political economy of the world trading system: the WTO and beyond. Oxford: Oxford University Press.

Hofmann, C., Osnago, A., Ruta, M. (2017). Horizontal Depth. A new database on the content of Preferential Trade Agreements. The World Bank, Policy Research Working Paper 7981.

Ikenberry, J. (2017). The plot against American Foreign Policy: Can the liberal order survive. Foreign Affairs, 96, 2-7.

Jawara, F., Kwa, A. (2003). Behind the scenes at the WTO: the real world of international trade publications. London: Zed Books.

Kahler, M. (1992). Multilateralism with Small and Large Numbers. En J. Ruggie, (Ed.), Multilateralism Matters. The theory and Praxis of an Institutional form. New York: Columbia University Press.

Keohane, R. (2006). The contingent legitimacy of multilateralism. In E. Newman, R. Thakur and J. Tirman (Eds.), Multilateralism Under Challenge: Power, International Order, And Structural Change. New York: United Nations University Press.

Low, P. (2011). WTO Decision-Making for the Future. WTO Staff Working Papers ERSD, 05. World Trade Organization (WTO), Economic Research and Statistics Division, available at https://www.wto.org/english/res_e/reser_e/ ersd201105_e.pdf

Martin, L. (1992). The Rational State Choice of Multilateralism. En J. Ruggie (Ed.), Multilateralism Matters. The theory and Praxis of an Institutional form. New York: Columbia University Press.

Martin, A., Mercurio, B. (2017). Doha dead and buried in Nairobi: Lessons for the WTO. Journal of International Trade Law and Policy, 16(1), 49-66. Disponible en http://dx.doi.org/10.1108/JITLP-01-2017-0001 
Mattoo, A., Subramanian, A. (2008). Multilateralism beyond Doha. The World Bank, Policy Research Working Paper 4735.

Méndez-Coto, M. (2016), Multilateralismo, gobernanza y hegemonía en la estructura económica internacional: Del G7 al G20. Relaciones Internacionales, $31,13-32$.

Narlikar, A. (2005). Bargaining over the Doha Development Agenda: Coalitions in the WTO. LATN Working Paper 36, available at http://latn.org.ar/wp-content/uploads/2015/01/wp-36.pdf

Neufeld, N. (2014). The long and winding road: How WTO members finally reached a Trade Facilitation Agreement. World Trade Organization, Economic Research and Statistics Division, Recuperado de https://www.wto.org/ english/res_e/reser_e/ersd201406_e.pdf

Obstfeld, M., and Taylor, A. (1997). The Great Depression as a Watershed: International Capital Mobility over the Long Run. NBER Working paper 5960.

OECD, OMC. (2015). Aid for Trade at a glance 2015. Reducing trade costs for inclusive sustainable growth. OECD Publishing. Available at http://dx.doi. org/10.1787/aid_glance-2015-en

OMC. (2011). Word Trade Report 2011. The WTO and preferential trade agreements: from co-existence to coherence. Ginebra: WTO press. Available at https:// www.wto.org/english/res_e/booksp_e/anrep_e/world_trade_report11_e.pdf

Pauwelyn, J., and Alschner, W. (2014). Forget about the WTO: The network of Relations between Preferential Trade Agreements (PTAs) and Double PTAs. Graduate Institute of International and Development Studies - Social Science Research Network. Available at, http://ssrn.com/abstract=2391124 or http://dx.doi.org/10.2139/ssrn.2391124

Primo Braga, C. (2017). The Threat of Economic Disintegration. In C. Primo Braga, and B. Hoekman, The future of the Global Trade Order. European University Institute.

Primo Braga, C., Hoekman, B. (2017). Future of the Global Trade Order. In C. Primo Braga, and B. Hoekman, The future of the Global Trade Order. European University Institute.

Rodrik, D. (2000). How far will international economic integration go? Journal of economic perspectives, 14(1), 177-186.

Rodrik, D. (2011). The globalization paradox. Oxford: Oxford University Press.

Rodrik, D. (2017). Straight talk on trade. New Jersey: Princeton University Press.

Rolland, S. (2010). Redesigning the negotiation process at the WTO. Journal of International Economic Law, 13(1), 65-110. 
Rosales, O., Herreros, S., Frohamann, A., and García-Millán, T. (2013). Las negociaciones megarregionales: Hacia una nueva gobernanza del comercio mundial. CEPAL, Serie Comercio Internacional n. ${ }^{\circ} 121$.

Stone, R. (2009). Institutions, Power and Interdependence. En H. Milner, A. Moravcsik, Power, Interdependence and Nonstate Actor in world politics. New Jersey: Princeton University Press.

Sanahuja, J. (2016). América Latina en un cambio de escenario: De la bonanza de las commodities a la crisis de la globalización. Revista Pensamiento Propio, 21(44), 13-26.

Sanahuja, J. (2018). Crisis de globalización, crisis de hegemonía: Un escenario de cambio estructural para América Latina y el Caribe. En A. Serbin (Ed.), América Latina y el Caribe frente a un nuevo orden mundial: Poder, globalización y respuestas regionales. Buenos Aires: Ediciones CRIES (Coordinadora Regional de Investigaciones Económicas y Sociales).

Steger, D. (2009). The future of the WTO: The case for institutional reform. Journal of International Economic Law, 12(4), 803-833.

Steinberg, F. (2007). Cooperación y conflicto. Comercio internacional en la era de la globalización. Madrid: Ed. AKAL.

Steinberg, R. (2002). In the Shadow of Law or Power? Consensus-Based Bargaining and Outcomes in the GATT/WTO. International Organization, 56(2), 339-374.

Vangrasstek, C. (2013). The History and Future of the World Trade Organization. Geneva: WTO Publications.

Vickers, B. (2013). The relationship between plurilateral approaches and the round. E15 Expert Group on Functioning of the WTO. ICTSD - World Economic Forum.

Wolfe, R. (2004). Informal Political Engagement in the WTO: Are Mini-Ministerials a Good Idea? In J. Curtis, D. Ciuriak (Eds.), Trade Policy Research 2004 Toronto: Minister of Public Works and Government Services - Canada.

Wolfe, R. (2009). The WTO single undertaking as negotiating technique and constitutive metaphor. Journal of International Economic Law, 12(4), 835-858.

Zelicovich, J. (2016). La economía política internacional en las negociaciones multilaterales de agricultura. El caso de las subvenciones a las exportaciones agrícolas en la Ronda Doha. Revista Estudios Sociales Contemporáneos. Instituto de Estudios Sociales Contemporáneos, 14, 64-84.

Zelicovich, J. (Mayo, 2017). ¿Está viva la OMC? Bloqueos y consensos en las negociaciones de las Conferencias Ministeriales de Bali y Nairobi. UNISCI Journal, 44. Universidad Complutense de Madrid.

Zurn, M. (2018). Contested Global Governance. Global Policy, 9(1), 138-145. 
